\title{
SIMULAÇÃO DE DESBASTE EM POVOAMENTO DE Hovenia dulcis MEDIANTE DENDROGRAMA DE MANEJO DE DENSIDADE
}

\author{
Gerson Luiz Selle ${ }^{1}$, Elisabete Vuaden ${ }^{2}$, Luiz Antônio Jacques de Albernard ${ }^{3}$, Evaldo Muñoz Braz ${ }^{4}$ \\ ${ }^{1}$ Eng. Florestal, Dr., Depto. de Ciências Florestais, UFSM, Santa Maria, RS, Brasil - hs@ smail.ufsm.br \\ ${ }^{2}$ Eng $^{\mathrm{a}}$ Florestal, Doutoranda em Eng. Florestal, UFSM, Santa Maria, RS, Brasil - elisabetevuaden@yahoo.com.br \\ ${ }^{3}$ Eng. Florestal, Santa Maria, RS, Brasil - albernardel@ hotmail.com \\ ${ }^{4}$ Eng. Florestal, Dr., Embrapa Florestas, Colombo, PR, Brasil - evaldo@cnpf.embrapa.br
}

Recebido para publicação: 05/06/2008 - Aceito para publicação: 05/02/2010

\begin{abstract}
Resumo
Este trabalho foi desenvolvido com o objetivo de estudar a densidade e simular intervenções, por meio de dendrogramas de manejo de densidade, em uma população de Hovenia dulcis localizada na região central do estado do Rio Grande do Sul. Para tanto, foram utilizados dados de diâmetro de copa e de fuste, coletados em três estratos: dominante, intermediário e dominado, pelo método da Densidade Pontual de Spurr, num povoamento de aproximadamente 1,3 hectares de uva do Japão com 25 anos de idade. Devido o local de coleta das informações apresentar grande variação na altura dominante, este foi dividido em Sítios I e II. Entre os estratos verificou-se que somente ocorreu diferença estatística significativa entre o estrato dominante do Sítio I e os demais de ambos os sítios. $\mathrm{O}$ coeficiente angular do ajuste das linhas de densidade ficaram estabelecidos, para o estrato dominante do Sítio I em -2,492 e, para os demais, em -1,085. A melhor forma de conduzir o povoamento seria aplicando um desbaste, se o mesmo fosse formado somente por árvores com dimensões semelhantes às do estrato dominante do Sítio I, e quatro intervenções se fosse constituído somente de indivíduos pertencentes aos demais estratos.

Palavras-chave: Sítio; modelo de Reineke; desbaste.
\end{abstract}

\begin{abstract}
Thinning simulation in Hovenia dulcis population trough density management dendrogram. The main objective of this study was to simulate density and interventions through density management dendrogram in a population of Hovenia dulcis located in the central region of the State of Rio Grande do Sul, Brazil. It was utilized Data from crown and stem diameters, collected in three types of tree stratum dominant, intermediate, and dominant, through the Punctual Density Method developed by Spurr, in a stand of approximately 1.3 hectares of Hovenia dulcis 25 years old were used. Due to the high variability in the trees dominant height the area was divided into two sites, I and II. The results showed that statistic significant difference was detected only between the dominant stratum of site I, and the other strata on both sites. The angular coefficients of the regression adjustment of the density lines were -2.492 for the site I dominant stratum and -1.085 for the others. It was also observed that the best way to manage the stand would be one thinning, if it was formed only by trees with similar dimensions of the dominant stratum of Site I, and four thinnings if the site was composed by individuals belonging to the other strata.

Keywords: Site; Reineke model; thinning.
\end{abstract}

\section{INTRODUÇÃO}

No cenário florestal atual, o meio científico tem importante papel a desempenhar, maximizando o rendimento das espécies atualmente plantadas e pesquisando novas espécies, de forma a aumentar o restrito grupo sobre o qual se tem o domínio do processo produtivo.

Sempre que se pensa em conduzir bem uma floresta, visando planejar e maximizar a produção madeireira, faz-se necessário saber o potencial produtivo do local.

Também é possível afirmar que, em um sítio, o espaço é um fator determinante, que controla a taxa de crescimento das árvores, descrita como densidade. Esta, definida pelo espaçamento entre as 
plantas, é provavelmente a principal técnica silvicultural aplicada em plantios florestais, sendo responsável pela qualidade da madeira e a produtividade, em função dos objetivos traçados para o plantio (SELLE; VUADEN, 2009).

Entre as medidas diretas de densidade, tem-se o número de árvores, que pode ser obtido de forma fácil e se relaciona diretamente com a idade, o sítio e o grau de utilização da área. O número de árvores pode variar sem afetar a densidade, o que depende do grau de ocupação do espaço físico pela área das copas e pelo sistema radicular das árvores.

A densidade que causa a morte das árvores é resultante da competição por água, luz e nutrientes, culminando com o domínio de algumas e a morte de outras. Essa supressão de indivíduos devido à competição extrema também é denominada de autodesbaste, que, ao mesmo tempo em que induz a um decréscimo exponencial da densidade, é responsável pelo aumento no tamanho dos indivíduos.

Para Schneider (1993) e Scheeren (2003), à medida que as árvores se desenvolvem, suas copas e raízes necessitam gradativamente de um maior espaço para continuar a crescer. Em caso de o espaço ser restrito, instala-se o processo de concorrência, ocorrendo a morte dos indivíduos pertencentes aos estratos inferiores.

A copa das árvores é seriamente afetada pela competição. Uma vez que a massa foliar é fator determinante no crescimento do câmbio, a dimensão da copa determina o crescimento do fuste das árvores (SPIECKER, 1981).

Assim sendo, Durlo (2001) salienta que a concorrência é o elemento que determina qual posição sociológica uma árvore ocupará no extrato de floresta, pois, tão logo se inicia essa concorrência entre as árvores, tem-se o fechamento do espaço aéreo pelas copas, começando a diferenciação entre as classes sociológicas, com alguns indivíduos se sobressaindo do dossel da floresta, enquanto outros são dominados por seus vizinhos. Além disso, também é conhecida a influência da proporção e vitalidade da copa sobre o crescimento diamétrico das árvores. Como consequência, as características morfométricas, que dependem de uma ou mais dessas variáveis, também serão determinadas pela posição sociológica dos indivíduos, tornando necessária a análise dessas relações, segregadas por estrato social. Para caracterizar as classes sociais das árvores, muitos pesquisadores propuseram diferentes formas de classificação sociológica, sendo a de Kraft uma das mais conhecidas.

Em florestas equiâneas, a diferenciação de seus indivíduos em estratos é iniciada quando o povoamento atinge o ponto de estágio denso, alterando a relação entre a altura e o diâmetro das árvores (h/d), sendo, portanto, essa relação influenciada pela posição sociológica das árvores (HUSCH et al., 1982).

Segundo Finger (1992), a relação h/d será maior ou menor dependendo do estrato da floresta a que a árvore pertence. Nas árvores dominantes, a altura é pouco afetada pela densidade, o mesmo não ocorrendo com as dominadas, em que a influência no crescimento é bastante pronunciada. A variável diâmetro do tronco é bastante afetada pela densidade em árvores de estratos inferiores. Dessa forma, quando se tem alta concorrência, a relação h/d será maior.

Reineke, em 1933, propõe que a relação entre o logaritmo do número de árvores por hectare e o logaritmo do diâmetro médio quadrático das árvores resulta numa linha reta e que o declive dessa reta poderia ser usado para definir os limites do estoque máximo ou o momento em que ocorre o autodesbaste (SPURR, 1952). Na prática, Reineke (1933) especifica que, quando se leva a um papel logaritmo, o número de árvores no eixo das ordenadas e o diâmetro médio no das abcissas, consegue-se uma relação linear entre essas variáveis. A reta resultante ajustada, que representa uma série de observações, indica o número de árvores por hectare para um povoamento de mesma idade em densidade completa.

Quanto a isso, para grande parte dos pesquisadores, a forma mais utilizada na determinação dessa linha é aquela em que as informações são levantadas sobre plantios com densidade completa, ocorrendo quando os indivíduos começam a morrer pela excessiva concorrência (falta de luz, nutrientes e água), a qual Peet; Christensen (1987) denominaram de desbaste natural ou autodesbaste. No entanto Chapmann; Meyer (1949) já salientavam que, na falta de povoamentos nessas condições, o melhor método para estimar a densidade em povoamentos equiâneos consiste na determinação do índice de densidade de Reineke, por ser independente da idade e da qualidade do sítio.

A quantidade e a qualidade da madeira dos povoamentos florestais estão, em grande parte, relacionadas com o sítio, tratos silviculturais e principalmente com a densidade da população. Sobre essa visão, é interessante considerar que, à medida que ocorre o aumento do número de árvores, também é induzido, de forma significativa, o aumento da área basal, até o momento em que a competição entre os 
indivíduos é iniciada, estabelecendo-se o início da competição extrema entre a população, com a manutenção ou redução da área basal.

Métodos mais específicos de medidas de densidade foram desenvolvidos para descrever o grau de concorrência em um determinado ponto de um povoamento. Essas medidas receberam a designação de densidade pontual e correlacionam o crescimento de uma árvore com a densidade de árvores em torno dela. O método de Spurr (1962) é um exemplo de uma técnica que fornece uma medida de densidade pontual e envolve a escolha de uma árvore ou ponto sobre o qual queremos determinar o grau de concorrência em torno das árvores, usando a teoria básica de Bitterlich da contagem angular da amostragem.

Estudos relacionados à elaboração de diagramas de manejo de densidade, denominados de DMDs, primeiramente elaborados por Ginrich (1967) para povoamentos florestais, são tidos como ferramentas úteis para administradores florestais, substituindo os tradicionais experimentos para definição de espaçamentos ideais. No entanto, pesquisas sobre o assunto ainda são muito escassas, principalmente para espécies folhosas. O trabalho elaborado pelo U.S. Forest Service (1970), para duas espécies de folhosas (Quercus e Carya), de Illinois, EUA, o de Smith; Gibbs (1970), para Acer sacchrum em cinco regiões dos EUA, e recentemente o de Gezan et al. (2007), com três espécies de Nothofagus (obliqua, alpina e dombeyi) no Chile, são os poucos exemplos encontrados na literatura. O número de estudos é menos raro quando se trata de espécies coníferas, caso em que se podem citar os trabalhos elaborados por Márquez-Linares; Alvarez-Zagoya (1995), para Pinus cooperi no México, o de Saunders; Puettmann (2000), para Picea glauca, que cresce na região norte dos EUA, e, recentemente, o de Schneider (2008), para Pinus taeda, na região central do estado de Santa Catarina, Brasil.

Para Reineke (1933), em povoamentos onde a densidade é completa, a relação entre o diâmetro médio quadrático das árvores e o número de árvores por unidade de área é linear (isso numa escala de duplo logaritmo). O autor também salienta que os parâmetros da equação são independentes da idade e do sítio e, por isso, podem ser empregados para estimar a densidade da população em função do diâmetro médio. Segundo ele, ao transpor para uma escala logarítmica a relação entre o número de árvores por unidade de área e o diâmetro médio quadrático, em povoamentos com densidade completa, tem-se uma linha reta, com um valor constante do coeficiente angular de -1,605, para qualquer espécie.

Já a lei do autodesbaste ou da potência do $-3 / 2$ de autodesbaste, desenvovida inicialmente para culturas anuais agrícolas, entre as décadas de 60 e 70, por pesquisadores japoneses (YODA et al., 1963), corresponde à descrição de uma linha formada pelo logaritmo do diâmetro, volume etc. da árvore média, com o logaritmo do número de árvores por unidade de área, em que ocorre um declive negativo de $-1,5$ ou -3/2. Um dos pioneiros a aplicar essa técnica nos Estados Unidos foi Ginrich (1967), mais tarde seguido por Drew; Flewelling (1979). Eles observaram que poderia haver relação entre o número máximo e o tamanho das árvores que compõem povoamentos de determinada espécie, com a sustentabilidade delas em qualquer local.

A correta regulagem da densidade é a chave para uma boa condução do povoamento em conformidade com os objetivos planejados, possibilitando a predição das condições futuras do povoamento. Isso só é possível devido à evolução dos modelos matemáticos ao longo das décadas e à incorporação dos conceitos biológicos, como a competição interespecífica, baseada em algumas das inúmeras relações de densidade e tamanho, que, por sua vez, referem-se às variáveis dendrométricas do povoamento, como o número de árvores e a área basal, entre outras. Diante do emprego dessas relações dendrométricas, torna-se possível construir importantes ferramentas para a condução de povoamentos florestais, como os dendrogramas da densidade. Em vista disso, o objetivo deste trabalho foi analisar a densidade por meio da copa de um povoamento de Hovenia dulcis Thunberg e simular desbastes sobre essa população com o auxílio de um diagrama de manejo de densidade.

\section{MATERIAL E MÉTODOS}

\section{Caracterização da espécie}

A árvore é conhecida vulgarmente com os nomes de uva-do-japão, uva-japonesa, banana-dojapão, caju-do-japão, mata-fome, pau-doce, chico-magro e passa-japonesa, e pertence à família das Rhamnaceae (CARVALHO, 1994). 
Ocorre naturalmente entre as coordenadas de $25^{\circ}$ a $41^{\circ}$ norte e de $100^{\circ}$ a $142^{\circ}$ leste de Greenwich, principalmente, na China, Japão e Coreias. É uma planta heliófila, caducifólia, alcançando alturas de 10 a $15 \mathrm{~m}$, podendo chegar até a $25 \mathrm{~m}$, com copa ampla. Seu diâmetro médio à altura do peito varia entre 20 e $40 \mathrm{~cm}$, podendo chegar a $50 \mathrm{~cm}$. Seu tronco geralmente é retilíneo e cilíndrico, apresentando fuste com até $8 \mathrm{~m}$ de comprimento, ramificação dicotômica, com ramos pubescentes enquanto jovem. Apresenta gemas dormentes subcorticais, rebrotando intensamente da touça, podendo ser manejada por talhadia, com rotações previstas de 10 a 15 anos (RIGATTO et al., 2001).

Nas Américas, a espécie vem sendo cultivada na Argentina, no Paraguai e no sul do Brasil, principalmente nas regiões de climas $\mathrm{Cfa}$, $\mathrm{Cfb}$ e $\mathrm{Cwa}$, de forma isolada ou em pequenos povoamentos. No sul do Brasil, são comuns pequenos plantios de uva-do-japão em propriedades agrícolas, implantados por mudas ou eventualmente por semeadura direta no terreno (RIGATTO et al., 2001).

A espécie é rústica, resistente ao ataque das formigas e geadas moderadas, não tendo problemas com pragas ou doenças que sejam limitantes ao seu desenvolvimento, crescendo bem em solos compactados, de média profundidade a profundos, excluindo-se aqueles com excesso de umidade. No meio rural, sua madeira é utilizada para lenha e moirões, com folhas e frutos empregados na alimentação do gado bovino. Além disso, sua flor é muito apreciada por abelhas, sendo considerada uma planta melífera, com frutos possuidores de propriedades medicinais (SELLE et al., 1993).

\section{Localização e caracterização das áreas de estudo}

O estudo foi realizado em uma população de Hovenia dulcis existente na Fundação Estadual de Pesquisa Agropecuária - Florestas (FEPAGRO), localizada sob as coordenadas geográficas de 2940'19" (latitude Sul) e 5354'03" (longitude Oeste), no município de Santa Maria, RS.

A população é formada por uma floresta plantada em 1983 e está em uma altitude média de $120 \mathrm{~m}$. O clima predominante, segundo a classificação de Köppen, é Cfa, com as temperaturas médias do mês mais frio entre $13{ }^{\circ} \mathrm{C}$ e $18{ }^{\circ} \mathrm{C}$, e as do mês mais quente superiores a $22{ }^{\circ} \mathrm{C}$. A precipitação pluviométrica é de $1.700 \mathrm{~mm}$, distribuídos regularmente durante todo o ano, com o maior número de dias com geada ocorrendo nos meses de junho e julho (MALUF, 2000).

\section{Caracterização dos locais de coleta}

O povoamento analisado, com área de 1,3 ha, foi estabelecido sobre um Argissolo Vermelho Distrófico Típico, pertencente à unidade de mapeamento São Pedro. São solos que apresentam o horizonte A espesso (em torno de $56 \mathrm{~cm}$ ), de cor bruno-escuro a bruno-avermelhado, franco argilo arenoso, com estrutura fraca em blocos subangulares e com transição clara e plana para o horizonte B.

O plantio avaliado é remanescente de um experimento onde foram testados 5 espaçamentos ( $4 \mathrm{~m}$ x $2 \mathrm{~m} ; 4 \mathrm{~m}$ x $3 \mathrm{~m} ; 4 \mathrm{~m}$ x $4 \mathrm{~m}$; $4 \mathrm{~m}$ x $5 \mathrm{~m}$ e $4 \mathrm{~m}$ x 6 m), com 4 repetições. Cada repetição compunha 7 linhas de plantas distanciadas 2 metros.

O terreno onde foi estabelecido o povoamento apresenta uma variação na declividade, refletindo no desenvolvimento da espécie, com a parte mais elevada tendo um melhor crescimento em altura. A parte mais baixa do terreno, por ter excesso de umidade nas camadas superficiais do solo, tem crescimento menor em altura.

Para este estudo, o experimento original foi considerado como uma população, sofrendo uma divisão por apresentar variação no fator sítio, identificado pela altura dominante de cada parcela. Para corrigir o problema, visando reduzir a variação interna da população, criou-se um novo arranjo para o povoamento, tendo como base a altura dominante (ASSMANN, 1970) calculada em cada parcela. Na nova estrutura, os Blocos foram substituídos por Sítio I e II, conforme tabela 1 e figura 1.

Como apresentavam poucas falhas e estas ocorrendo principalmente nos menores espaçamentos, as árvores estavam submetidas a diferentes níveis de competição, com a ocorrência de sobreposição de copas.

O sub-bosque é caracterizado por uma intensa regeneração natural da espécie principal, principalmente onde os espaçamentos são maiores e a altura das árvores é menor, possibilitando a entrada de grandes quantidades de luz pelo dossel. Também pode ser verificada a presença, no sob-bosque, de um significativo número de outras espécies, como o cedro, a canela, o camboatá-vermelho, o carvalhinho, o chal-chal, a mamica-de-cadela e a pitangueira, entre outras. Todos esses aspectos podem ser visualizados na figura 2. 
Tabela 1. Reestruturação do experimento, dividido em dois sítios, com base na altura dominante de Assmann, de cada parcela de Hovenia dulcis.

Table 1. Restructuring of the experiment, divided into two sites, based on the dominant height of Assmann, of each parcel of Hovenia dulcis.

\begin{tabular}{lccc}
\hline $\begin{array}{l}\text { Arranjo original } \\
\text { Bloco }\end{array}$ & $\begin{array}{c}\text { Arranjo novo } \\
\text { Sítio }\end{array}$ & Espaçamentos (m) & $\mathbf{h}_{\mathbf{1 0 0}}(\mathbf{m})$ \\
\hline $\mathrm{B}_{1}$ & I & $4 \times 3$ & 22,6 \\
$\mathrm{~B}_{1}$ & I & $4 \times 6$ & 22,0 \\
$\mathrm{~B}_{1}$ & I & $4 \times 5$ & 23,2 \\
$\mathrm{~B}_{1}$ & I & $4 \times 4$ & 22,9 \\
$\mathrm{~B}_{1}$ & I & $4 \times 2$ & 20,6 \\
$\mathrm{~B}_{2}$ & I & $4 \times 5$ & 22,4 \\
$\mathrm{~B}_{2}$ & I & $4 \times 3$ & 22,7 \\
$\mathrm{~B}_{2}$ & I & $4 \times 4$ & 24,0 \\
$\mathrm{~B}_{2}$ & I & $4 \times 6$ & 22,1 \\
$\mathrm{~B}_{2}$ & II & $4 \times 2$ & 17,9 \\
$\mathrm{~B}_{3}$ & II & $4 \times 6$ & 19,7 \\
$\mathrm{~B}_{3}$ & I & $4 \times 4$ & 22,6 \\
$\mathrm{~B}_{3}$ & I & $4 \times 2$ & 23,1 \\
$\mathrm{~B}_{3}$ & II & $4 \times 5$ & 20,2 \\
$\mathrm{~B}_{3}$ & II & $4 \times 3$ & 16,6 \\
$\mathrm{~B}_{4}$ & II & $4 \times 3$ & 19,1 \\
$\mathrm{~B}_{4}$ & II & $4 \times 2$ & 18,9 \\
$\mathrm{~B}_{4}$ & II & $4 \times 4$ & 17,4 \\
$\mathrm{~B}_{4}$ & II & $4 \times 5$ & 17,3 \\
$\mathrm{~B}_{4}$ & II & $4 \times 6$ & 17,5 \\
\hline
\end{tabular}

$\mathrm{h}_{100}$ : altura dominate de Assmann, em metros.
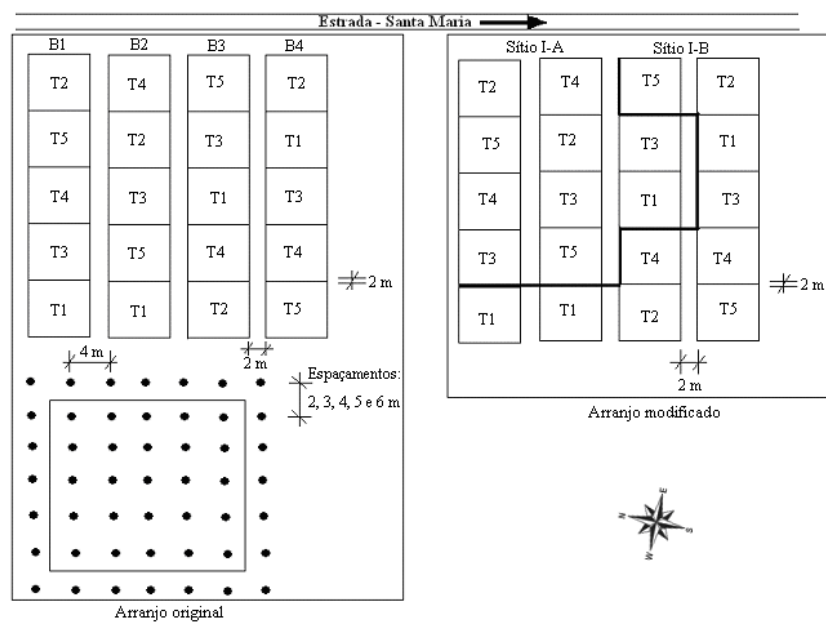

Figura 1. Arranjo inicial e nova ordenação da população de Hovenia dulcis.

Figure 1. Original arrangement and ordering of new population of Hovenia dulcis.

\section{Coleta dos dados}

Originalmente, o povoamento era dividido em blocos e parcelas, sendo cada parcela correspondente a um tratamento que continha 49 árvores. Os blocos e parcelas (tratamentos) eram separados por uma distância de $2 \mathrm{~m}$. Além disso, cada parcela tinha uma bordadura composta de uma fileira de árvores na coluna e na linha, perfazendo 25 árvores cada.

A coleta dos dados foi realizada sob árvores pertencentes a três estratos. A classificação foi baseada na relação h/d, onde a altura é medida em metros e o diâmentro em centímetros. Os estratos tiveram as seguintes definições: 
a) estrato dominante - composto por árvores cuja relação $\mathrm{h} / \mathrm{d}$ assumisse valores menores que 1,0 .

b) estrato intermediário - a relação deveria ser igual a 1,0.

c) estrato dominado - composto por árvores que apresentassem valores superiores a 1,0.

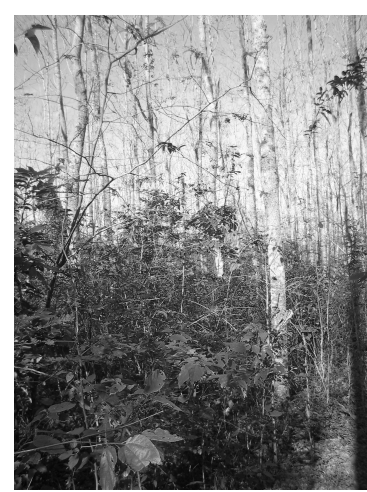

Figura 2. Aspecto do sub-bosque do povoamento de Hovenia dulcis, com 25 anos.

Figure 2. Aspect of the sub-population of the forest Hovenia dulcis, with 25 years.

Essa diferenciação foi estabelecida com base na condição de que uma árvore, para pertencer ao estrato dominante, quando em condições de concorrência, num povoamento, possui altura maior em relação às pertencentes aos demais estratos. Isso porque, em povoamentos submetidos à concorrência, as árvores desenvolvem-se para atingirem o dossel, em busca da luz, provocando um maior aumento da dimensão altura em relação ao diâmetro, tendo uma relação h/d menor. Já as árvores que não conseguem atingir o estrato dominante (intermediárias e dominadas), devido à escacez de luz, não atingem grandes alturas, mas conseguem crescer em diâmetro, devido à presença de copas grandes, tendo uma relação h/d maior quanto maior for o grau de concorrência sofrido.

Para a determinação do número de árvores e a área basal, utilizou-se a metodologia apresentada por Spurr (1962), denominada de densidade pontual. Segundo o autor, o método é um dos mais indicados para avaliar a densidade em pontos individuais dentro de um povoamento. Utiliza um ângulo fixo e predeterminado, muito semelhante ao método de Bitterlich. Por esse método, todas as árvores maiores que esse ângulo são incluídas na unidade amostral. Todos os indivíduos próximos à árvore escolhida como central são avaliadas, para verificação da sua inclusão ou não na unidade amostral. Essa avaliação é baseada no FAB (fator de área basal) de 2,3, proposto por Daniels et al. (1986), para identificar árvores competidoras.

A ordem de inclusão das árvores competidoras na parcela foi realizada mediante um ranqueamento entre o diâmetro da árvore em análise e a distância desta até a árvore central. Com isso, quanto maior fosse essa relação, maior o ângulo criado entre a árvore central e as tangentes da árvore em análise, indicando maior influência, sendo esse processo repetido com todas as árvores próximas à árvore central. Ao identificar que a primeira árvore participou da amostra, foi encontrado um ângulo, que formou a primeira área de amostragem, e com isso se fez a primeria estimativa de área basal. Prosseguindo, o segundo ângulo foi encontrado para a segunda árvore, formando-se a segunda área de amostragem, a partir da qual foi realizada a segunda estimativa de área basal. Assim, repetiu-se o processo até que todas as árvores competidoras fossem incluídas na parcela. A estimativa final da área basal foi a média das estimativas individuais. Para Spurr (1962), esse método é naturalmente mais preciso que qualquer outro de estimativa da área basal pontual.

Para a determinação da densidade pontual, foram coletadas informações de 16 árvores pontuais, em cada uma das três posições sociológicas, totalizando 48 árvores pontuais e 153 árvores vizinhas.

\section{Linhas de ocupação dos estratos do povoamento}

A linha é uma forma de verificação da competição em que se encontra o povoamento, nos três estratos (dominante, intermediário e dominado) dos dois locais (Sítio I e II). 
Com a melhor relação entre o diâmetro de copa e o diâmetro do fuste, definida por meio de correlações de Pearson, foram comparados, por análise de covariância, os estratos e os sítios, verificandose as tendências e os níveis entre essas possíveis fontes de variação. Conforme a existência ou não de diferenças entre as fontes de variação testadas, foram definidos grupos de dados semelhantes, por meio da projeção da ocupação do local pelo diâmetro das copas, relacionados com o respectivo diâmetro do fuste, definindo a densidade de árvores necessárias para preencher o espaço correspondente a uma unidade de área (equação 1). Para cada grupo de dados semelhantes, foi ajustado um modelo de Reineke (equação 2) que definirá a linha de densidade de ocupação do respectivo estrato ou grupo de estratos.

$$
N / h a=\frac{10000 \times 4}{\pi d c^{2}}
$$

Em que: $d c=$ diâmetro de copa em metros; $\pi=$ constante cujo valor é 3,141593.

$$
N=\alpha d^{-\beta}
$$

Em que: $\mathrm{N}$ = número de árvores por hectare; " $\alpha$ ” e " $\beta$ " = parâmetros; " $d$ " = diâmetro.

Para ajustar as regressões lineares, foram utilizados procedimentos do pacote Statistical Analysis System (SAS, 1999), e para os demais cálculos foi usada a planilha eletrônica Excel (Microsoft ${ }^{\circ}$ Oficce, 2003).

\section{RESULTADOS E DISCUSSÃO}

\section{Linhas de ocupação da população}

Para definir a melhor relação entre o diâmetro de copa (dc) e o diâmetro do fuste (DAP), primeiramente efetuou-se um teste de correlação de Pearson (Tabela 2).

Tabela 2. Correlações de Pearson entre as variáveis diâmetro da copa (dc) e diâmetro do fuste (DAP), de Hovenia dulcis, nos diferentes estratos fitossociológicos e locais (Sítio I e II).

Table 2. Pearson's correlations between crown diameter (dc) and diameter at breast height (DBH) of

\begin{tabular}{|c|c|c|c|c|c|c|}
\hline \multirow{2}{*}{$\begin{array}{l}\text { Variável } \\
\text { independente }\end{array}$} & \multicolumn{6}{|c|}{ Variável dependente } \\
\hline & dc & $\ln (d c)$ & $1 / \mathrm{dc}$ & $\mathrm{dc}^{2}$ & $\overline{\sqrt{d c}}$ & $d c^{3}$ \\
\hline \multirow{2}{*}{ dap } & 0,81510 & 0,81676 & 0,79957 & 0,78999 & 0,81926 & 0,74836 \\
\hline & $<0,0001$ & $<0,0001$ & $<0,0001$ & $<0,0001$ & $<0,0001$ & $<0,0001$ \\
\hline \multirow{2}{*}{ 1/dap } & 0,69874 & 0,73221 & 0,74201 & 0,64929 & 0,71797 & 0,59137 \\
\hline & $<0,0001$ & $<0,0001$ & $<0,0001$ & $<0,0001$ & $<0,0001$ & $<0,0001$ \\
\hline \multirow{2}{*}{$1 / \mathrm{dap}^{2}$} & 0,60069 & 0,79079 & 0,78220 & 0,54631 & 0,62426 & 0,48776 \\
\hline & $<0,0001$ & $<0,0001$ & $<0,0001$ & $<0,0001$ & $<0,0001$ & $<0,0001$ \\
\hline \multirow{2}{*}{$\ln ($ dap) } & 0,77212 & 0,79079 & 0,78220 & 0,73307 & 0,78447 & 0,68117 \\
\hline & $<0,0001$ & $<0,0001$ & $<0,0001$ & $<0,0001$ & $<0,0001$ & $<0,0001$ \\
\hline
\end{tabular}
Hovenia dulcis, located in different phytosociologic stratum and sites (Site I and II).

$d c$ : diâmetro de copa (m); DAP: diâmetro do fuste medido a 1,30 $\mathrm{m}$ do solo $(\mathrm{cm}) ; \ln$ : logaritmo neperiano.

Das correlações apresentadas na tabela 2, as melhores são representadas por $\sqrt{d c}=f($ dap $)$, $\ln (d c)=f(d a p)$ e $d c=f(d a p)$, com valores muito semelhantes e altamente significativos. Foi selecionada a relação mais simples, $d c=f(d a p)$.

A partir da definição da melhor relação entre o diâmetro da copa e o diâmetro do fuste, procedeu-se à análise de covariância, para testar a existência ou não de diferença em inclinação (tendência) entre os diâmetros de copa nos diferentes sítios e estratos, sendo os resultados expressos na tabela 3.

A análise da tabela 3 demonstra que, para o teste de inclinação, sob um nível de significância de $5 \%$ de probabilidade de erro, tanto no Sítio I como no Sítio II, o diâmetro da copa do estrato dominante difere significativamente do intermediário, o mesmo acontecendo com as dominantes e dominadas do Sítio I. Porém as copas de árvores dominantes localizadas no Sítio II não diferem significativamente das pertencentes ao estrato intermediário do mesmo sítio. Essa não-diferenciação é pertinente, devido a fatores que limitam o crescimento nesse sítio, fazendo com que ocorra uma menor diferenciadção na amplitude, principalmente entre essas classes de sítio. 
Tabela 3. Análise de covariância, para teste de tendência, do modelo dc $=f(\mathrm{dap})$, entre os sítios e estratos de Hovenia dulcis.

Table 3. Analysis of covariance, test for trend, of model $\mathrm{dc}=f(\mathrm{dap})$, between sites and strata of Hovenia dulcis.

\begin{tabular}{lccccc}
\hline FV & GL & SQ & QM & F & Prob. > F \\
\hline Modelo & 9 & 219,56 & 24,40 & 54,53 & $<0,0001$ \\
DAP & 1 & 41,95 & 41,98 & 93,85 & $<0,0001$ \\
S & 1 & 2,38 & 2,38 & 5,33 & 0,0220 \\
E & 2 & 7,63 & 3,82 & 8,53 & 0,0003 \\
DAP*E & 1 & 0,37 & 0,37 & 0,84 & 0,3618 \\
DAP*S*E & 4 & 11,60 & 2,90 & 6,48 & $<0,0001$ \\
Resíduo & 193 & 86,34 & 0,47 & & PR > t $\mid$ \\
\hline Total & 202 & 305,91 & & Vt & 0,0005 \\
\hline Parâmetro & & Estimativa & Erro & 3,56 & $<, 0001$ \\
\hline DAP*SI*E1 x DAP*SI*E2 & 0,158171497 & 0,04437486 & 0,49 & $<, 3525$ \\
DAP*SI*E1 x DAP*SI*E3 & 0,194782581 & 0,04342078 & 4,20 & $<0,0001$ \\
DAP*SI*E2 x DAP*SI*E3 & 0,036611083 & 0,03927981 & $-1,17$ & 0,2418 \\
DAP*SII*E1 x DAP*SII*E3 & 0,201099531 & 0,04791511 & 1,17 & 0,2418 \\
DAP*SII*E1 x DAP*SII*E2 & $-0,056540868$ & 0,04815788 & 0,04815788 & \\
DAP*SII*E2 x DAP*SII*E3 & 0,056540868 & & & \\
\hline
\end{tabular}

FV: fonte de variação; GL: graus de liberdade; SQ: soma dos quadrados; QM: quadrado médio; F: valor de F calculado para a variável dependente; Prob.>F: nível de probabilidade de erro; dap: diâmetro medido a 1,30 m do solo; SI: sítio I; SII: sítio II; E: estratos (1: dominante, 2: intermediário e 3: dominado); Vt: valor da estatística $t$.

Árvores localizadas nos estratos intermediários e inferiores, por sua vez, não diferem estatisticamente.

Dessa forma, procedeu-se a uma nova análise de covariância, para verificar a existência ou não de igualdade, em tendência e nível, dos diâmetros de copa das árvores em função dos diâmetros do fuste, dos estratos dominante, intermediário e dominado de ambos os sítios (Tabela 4).

Considerando uma significância de 5\% do erro probabilístico, a tabela 4 demonstra a nãoocorrência de diferença significativa, em inclinação, entre os estratos dominantes dos sítios I e II. Assim sendo, faz-se necessária uma nova análise, para verificação do nível entre os estratos dos diferentes locais. Constatou-se a ocorrência de diferenças significativas, em nível, entre os estratos dominantes dos dois sítios. Além disso, o coeficiente angular $(-0,59340117)$ informa que as copas das árvores do Sítio II são, aproximadamente, meio metro menores que as do Sítio I. Essa situação pode estar condicionada aos fatores desfavoráveis oferecidos pelo Sítio II, relacionados ao excesso de umidade presente em camadas inferiores do solo, provocando baixa estatura e diâmetros das árvores, e à concorrência por espaço aéreo, em busca de luz, condicionando a formação de copas relativamente maiores.

As copas das árvores dos estratos intermediários e dominados, de ambos os sítios, não apresentaram diferença significativa em inclinação. Por isso, para fins de análise, foram considerados iguais e unidos, e uma nova análise de covariância foi realizada, agora testando a inclinação entre a união dos estratos intermediários e dominados, dentro de cada um dos sítios.

Para um nível de significância do erro probabilístico de 5\%, os diâmetros de copa das árvores pertencentes à união dos estratos intermediários e dominados dos sítios I e II demonstraram diferenciação significativa em inclinação.

A projeção gráfica das funções ajustadas para cada um dos estratos, em seus respectivos sítios, é apresentada na figura 3 .

Analisando a figura 3, observa-se que as árvores pertencentes à união dos estratos dominado e intermediário do Sítio I apresentam a tendência diferenciada do Sítio II, porém em média os valores são muito semelhantes. Já o estrato dominante do Sítio I apresenta valores consideravelmente superiores aos demais, e isso identifica que a população estudada é constituída de dois grupos distintos, um formado pelas árvores ocupantes do estrato dominante do Sítio I, que apresentam diâmetros do fuste (dap) consideravelmente maiores para um mesmo diâmetro de copa, e outro formado pelos demais estratos de ambos os sítios. 
Tabela 4. Análise de covariância do modelo dc $=f($ dap), entre os estratos dos Sítios I e II de Hovenia dulcis.

Table 4. Covariance analysis for model $\mathrm{dc}=f(\mathrm{dap})$, between the strata of sites I and II of Hovenia dulcis.

\begin{tabular}{|c|c|c|c|c|c|}
\hline FV & GL & SQ & QM & $\mathbf{F}$ & Prob. > F \\
\hline \multicolumn{6}{|c|}{ Teste para tendência entre os sítios I e II - estratos dominantes } \\
\hline Modelo & 3 & 39,14 & 13,05 & 23,73 & $<0,0001$ \\
\hline DAP & 1 & 35,21 & 35,21 & 64,03 & $<0,0001$ \\
\hline S & 1 & 0,23 & 0,23 & 0,42 & 0,5169 \\
\hline DAP x S & 1 & 0,10 & 0,10 & 0,18 & 0,6704 \\
\hline Resíduo & 88 & 48,39 & 0,55 & & \\
\hline Total & 91 & 87,53 & & & \\
\hline Parâmetro & & Estimativa & Erro & Vt & $\mathbf{P R}>|\mathbf{t}|$ \\
\hline dap*SIE1 $x$ dap*SII & & 0,049987226 & 0,11703514 & 0,43 & 0,6704 \\
\hline \multicolumn{6}{|c|}{ Teste para nível entre os sítios I e II - estratos dominantes } \\
\hline FV & GL & SQ & $\mathrm{QM}$ & $\mathrm{F}$ & Prob. > F \\
\hline Modelo & 2 & 39,04 & 19,52 & 35,83 & $<0,0001$ \\
\hline dap & 1 & 35,21 & 35,21 & 64,63 & $<0,0001$ \\
\hline $\mathrm{S}$ & 1 & 3,27 & 3,27 & 6,00 & 0,0163 \\
\hline Resíduo & 89 & 48,49 & 0,45 & 0,54 & \\
\hline Total & 91 & 87,53 & & & \\
\hline Parâmetro & & Estimativa & Erro & Vt & $\mathbf{P R}>|\mathbf{t}|$ \\
\hline dap*SI x dap*SII & & $-0,59340117$ & 0,24226630 & $-2,45$ & 0,0163 \\
\hline \multicolumn{6}{|c|}{ Teste para tendência entre os sítios I e II - estratos intermediários + dominados } \\
\hline FV & GL & SQ & QM & $\mathrm{F}$ & Prob. $>\mathrm{F}$ \\
\hline Modelo & 3 & 33,54 & 11,18 & 30,79 & $<0,0001$ \\
\hline dap & 1 & 22,61 & 22,61 & 62,28 & $<0,0001$ \\
\hline $\mathrm{S}$ & 1 & 3,71 & 3,71 & 10,21 & 0,0018 \\
\hline dap x S & 1 & 4,04 & 4,04 & 11,13 & 0,0012 \\
\hline Resíduo & 107 & 38,85 & 0,36 & & \\
\hline Total & 110 & 72,39 & & & \\
\hline Parâmetro & & Estimativa & Erro & Vt & $\mathbf{P R}>|\mathbf{t}|$ \\
\hline dap*SI x dap*SII & & $-0,11393440$ & 0,03415632 & $-3,34$ & 0,0012 \\
\hline
\end{tabular}

FV: fonte de variação; GL: graus de liberdade; SQ: soma dos quadrados; QM: quadrado médio; F: valor de F calculado para a variável dependente; Prob.>F: nível de probabilidade de erro; dap: diâmetro medido a 1,30 m do solo; SI: sítio I; SII: sítio II; E1: estrato 1 (dominante); Vt: valor da estatística $t$.

Proporcionalmente, as árvores dominantes do Sítio I apresentam copas menores, para uma mesma altura considerada (estrato), quando comparadas com as demais, evidenciando uma maior eficiência destas.

Trabalho semelhante foi desenvolvido por U.S. Forest Serviçe (1970), estudando a relação diâmetro de copa em função do diâmetro do tronco de Quercus e Carya, situadas em sítios bons e médios e em três estratos distintos (dominante, codominante e intermediário). Porém os autores concluíram que não houve diferença no diâmetro das copas entre as espécies, os sítios e os estratos avaliados, e que a relação diâmetro de copa e diâmetro do fuste foi próxima a linear.

Assim, os dados foram divididos em dois grupos, e para estimar o número de árvores necessário para a completa ocupação do espaço de um hectare, foi utilizada a equação 1 em cada um dos grupos de dados. Sobre eles se ajustou o modelo de Reineke (equação 2), proporcionando a obtenção da linha que descreve a ocupação das árvores do estrato dominante do Sítio I, cujo coeficiente angular é $-2,492$, e da união dos demais estratos (dominado + intermediário, dos sítios I e II, acrescido do dominante do Sítio II), com o coeficiente angular de -1,085. A projeção gráfica dessas duas retas é apresentada na figura 4.

Para a mesma área e espécie, Selle (2009) estabeleceu para linha de autodesbaste (A) o modelo $\mathrm{N} / \mathrm{ha}=100 \cdot \mathrm{d}^{-1,5}$, e para linha de fechamento do dossel $(\mathrm{D})$, a função $\mathrm{N} / \mathrm{ha}=26 . \mathrm{d}^{-1,5}$. Ao se plotarem essas linhas sobre os dados observados nos sítios I e II (estratos dominante, intermediário e dominado) e traçar 
limites, inferior e superior, de ocupação dessa população, obtiveram-se interessantes resultados, conforme mostra a figura 5, em escala logarítmica.

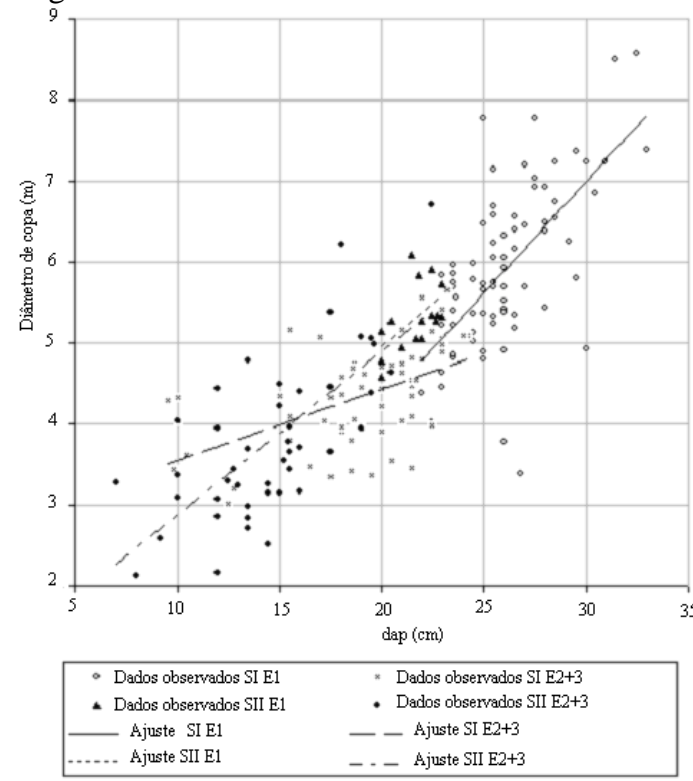

Figura 3. Ajustes dos diâmetros de copa (dc) em função dos diâmetros do fuste (dap) para os estratos e sítios de Hovenia dulcis.

Figure 3. Adjustment of the crown diameter (dc) as a function of stem diameters (dbh) for the strata and sites of Hovenia dulcis.

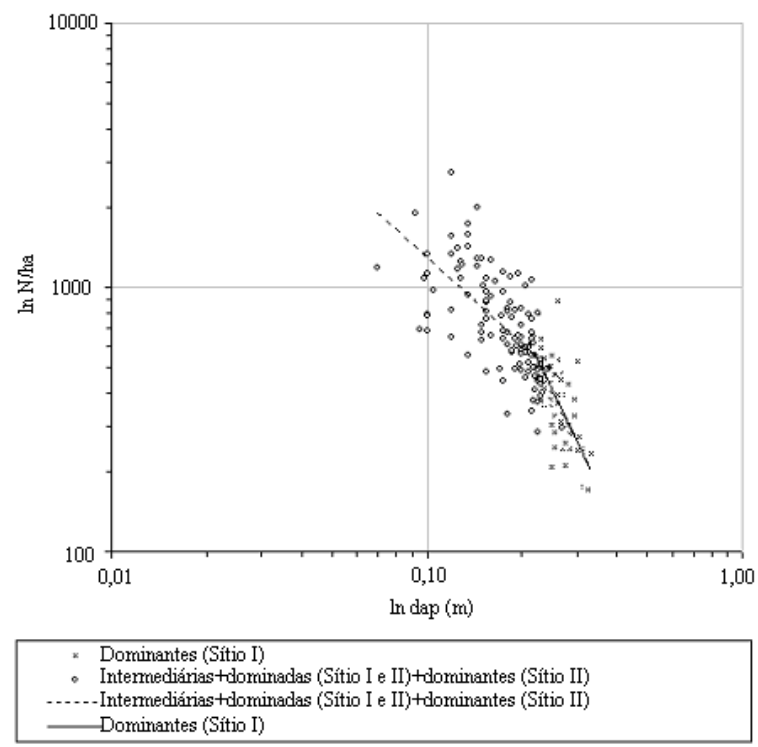

Figura 4. Ajuste de modelos que expressam as linhas de ocupação para os estratos dominante (Sítio I) e a união do dominado + intermediário (sítios I e II) + dominante (Sítio II), de Hovenia dulcis, em escala logarítmica.

Figure 4. Adjustment of models that express the lines of occupation, for the dominant strata (Site I) and the union dominated + intermediary (Sites I and II) + dominant (Site II) of Hovenia dulcis in logarithmic scale. 


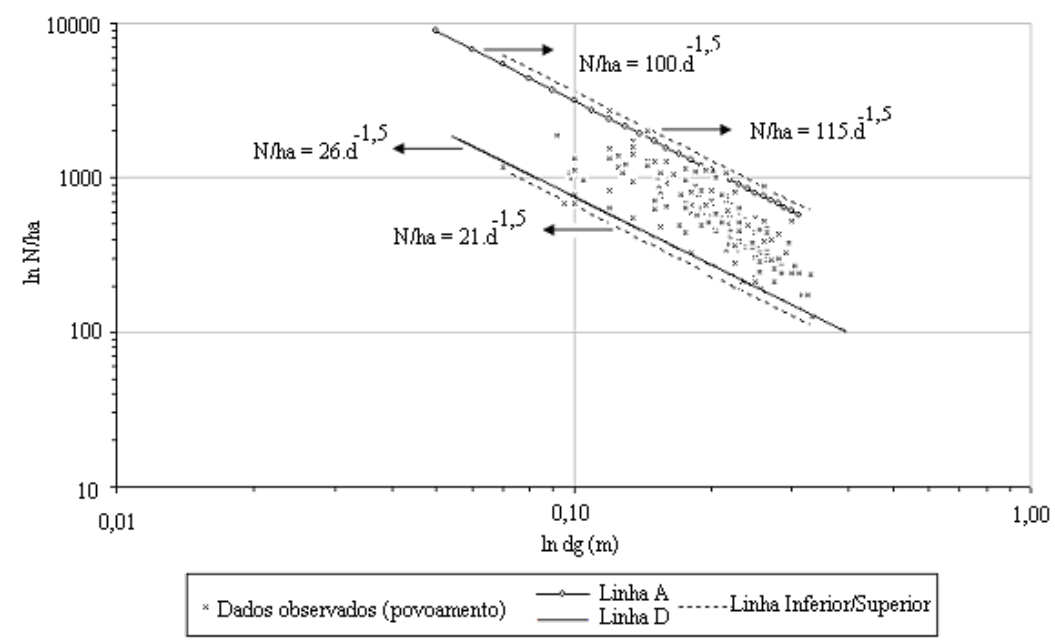

Figura 5. Limite inferior e superior da ocupação dos sítios, linhas de fechamento do dossel e autodesbaste, de Hovenia dulcis, em escala logarítmica.

Figure 5. Lower and upper limits of the occupation of sites, lines of closing canopy and self thinning of Hovenis dulcis in logarithmic scale.

A figura 5 apresenta a linha que identifica o limite inferior de ocupação do local (linha tracejada inferior) com um traçado pouco abaixo da linha D. As árvores que margeiam essa faixa possuem características muito semelhantes às árvores livres, possuindo copas relativamente grandes, de baixa estatura, submetidas a uma pequena densidade, possuindo, por isso, uma baixa eficiência no uso do local, localizadas em espaçamentos amplos, principalmente do Sítio II.

Já na parte superior do gráfico podem ser visualizadas as linhas A (autodesbaste) e do limite superior de ocupação do local (linha tracejada). Nota-se que ambas apresentam trajetórias muito semelhantes, o que identifica que as árvores do povoamento localizadas nessa faixa já estariam em elevada densidade, com apresentação de copas pequenas, portanto eficientes. As árvores localizadas nessa faixa estão no estrato dominante, principalmente do Sítio I.

Segundo a literatura, a capacidade de suportar uma maior ou menor densidade está diretamente relacionada com o local (sítio) e também com a espécie. Uma referência pertinente ao assunto é o trabalho desenvolvido por Gezan et al. (2007), que estudaram três gêneros de Nothofagus, espécie nativa do Chile, com a conclusão de que a espécie dombeyi suporta densidades mais altas em relação às espécies obliqua e alpina. Segundo eles, a diferença entre os locais poderia ser o fator determinante.

\section{Simulação de desbastes auxiliada pelo diagrama de manejo}

Como foi observada na população a existência de dois grupos distintos de árvores, as pertencentes ao estrato dominante do Sítio I e as pertencentes aos demais estratos dos sítios I e II, serão feitas duas simulações: no primeiro caso, imaginando que o povoamento esteja composto somente com árvores semelhantes às dominantes, e, no segundo caso, com uma população formada por árvores semelhantes aos demais estratos que compõem os sítios I e II.

Para simular os desbastes, foi utilizado o dendrograma para manejo da densidade de uva-dojapão elaborado por Selle (2009).

Cabe salientar que, nas duas situações propostas, o povoamento a ser considerado não foi implantado com os espaçamentos das simulações. Também deve ser considerado que a espécie apresenta características propensas à emissão de galhos epicórnicos quando ocorre uma grande inciência de luz no fuste, o que leva à desvalorização da madeira, se destinada à serraria, por apresentar o tronco impregnado de nós. Sendo assim, não deve haver intervenções, em populações dessa espécie, com abertura excessiva no dossel, devido aos fatores já citados, além da propenção à incidência de ventos que possam vir a ocasionar danos.

Os índices de sítio sobre os quais está acentado o povoamento vão de 20 a 24 metros, segundo classificação elaborada por Selle (2009) para a área e espécie. Para a primeira situação, o povoamento foi 
composto somente por árvores com dimensões iguais às dominantes encontradas no Sítio I. A linha ajustada para essa condição (Figura 6) demonstra um diâmetro da árvore de área basal média (dg) de $22 \mathrm{~cm}$, uma ocupação de 563 árvores por hectare, aos 25 anos de idade. Portanto cada indivíduo ocupa uma área aproximada de $18 \mathrm{~m}^{2}$, equivalendo a um espaçamento regular entre árvores de 4 x 4,5 m.

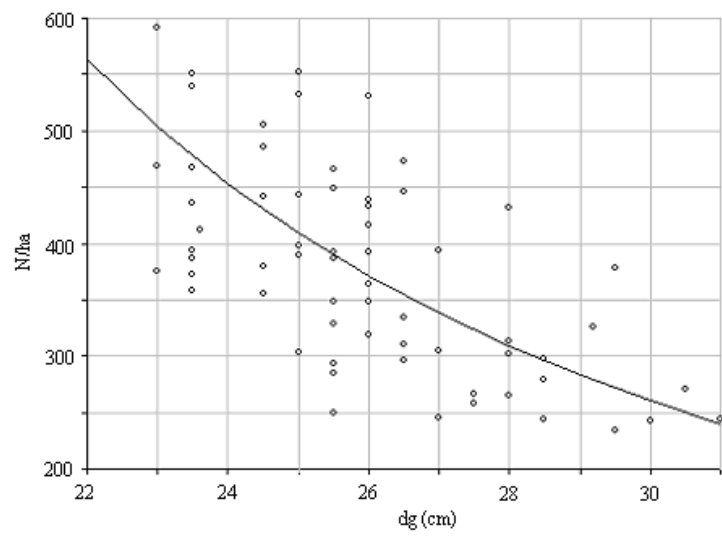

- Dados observados no estrato dominante (Sítio I) - Ajuste para estrato dominante (Sítio I)

Figura 6. Linha de densidade de árvores observadas em um povoamento de Hovenia dulcis com 25 anos, pertencente ao estrato dominante da população.

Figure 6. Line density of trees found in a stand of Hovenia dulcis at 25 years, belonging to the dominant stratum of the population.

Nesse caso, é aconselhado intervir aplicando apenas um desbaste antes do corte raso, no final da rotação. A demonstração planejada do número de árvores remanescentes e a respectiva área basal, assim como o que será retirado na intervenção e seu peso, constam na tabela 5.

Tabela 5. Plano de aplicação de um desbaste na condução de um povoamento de Hovenia dulcis com densidade de 563 árvores/ha, em local com índice de sítio de 20 a 24 metros e idade de 25 anos.

Table 5. Plan of implementation of a thinning in the conduct of a stand of Hovenia dulcis with density of 563 trees/ha on site with site index of 20 to 24 meters and 25 years of age.

\begin{tabular}{|c|c|c|c|c|c|c|}
\hline \multirow{2}{*}{$\operatorname{dg}(\mathrm{cm})$} & \multicolumn{2}{|c|}{ Remanescente } & \multicolumn{4}{|c|}{ Desbaste } \\
\hline & N/ha & $\mathbf{G}\left(\mathrm{m}^{2} / \mathrm{ha}\right)$ & N/ha & Peso (\%N/ha) & G $\left(\mathrm{m}^{2} / \mathrm{ha}\right)$ & Peso (\%G) \\
\hline 22 & 563 & 21,40 & & & & \\
\hline 26 & 340 & 18,05 & 223 & 39,61 & 3,35 & 15,65 \\
\hline 31 & 340 & 25,66 & & & & \\
\hline
\end{tabular}

dg: diâmetro de área basal média; N/ha: número de árvores por hectere; G: área basal por hectare; \%G: percentual da área basal por hectare.

De acordo com o proposto, o povoamento será conduzido, a partir da idade de 25 anos, com a aplicação de apenas um desbaste de intensidade moderada, retirando-se 15,6\% da área basal, com o corte raso no final do ciclo. Em termos de número de árvores na intervenção, serão retiradas $39,6 \%$. Graficamente, a demonstração é apresentada na figura 8.

Para a segunda situação, em que o povoamento está composto somente de árvores com dimensões iguais aos estratos dominado e intermediário dos sítios I e II mais indivíduos que compõem o estrato dominante do Sítio II, a linha ajustada para essa condição (Figura 7) demonstra o diâmetro da árvore de área basal média $(\mathrm{dg})$ de $7 \mathrm{~cm}$, uma densidade de 1917 árvores por hectare, aos 25 anos de idade. Com isso, cada árvore ocupa uma área média aproximada de $5,2 \mathrm{~m}^{2}$, o que equivale a um espaçamento regular entre indivíduos de 2 x 2,6 m. 


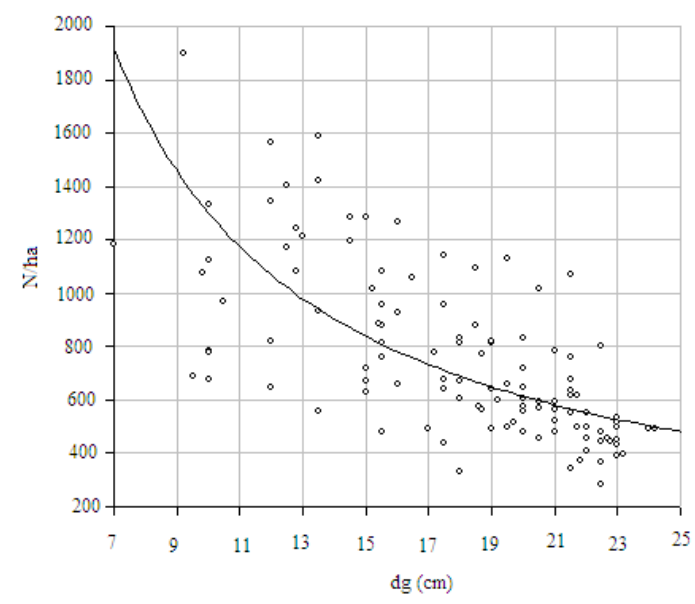

- Dados observados nos estratos dominado-interme diário (Sitios I e II)-doinante (Sitio II
— Ajuste para os estratos dominado-intermediário (Sitio I e II)-dominante (Sitio II)

Figura 7. Linha de densidade de árvores observadas em um povoamento de Hovenia dulcis com 25 anos, pertencente aos estratos dominado + intermediário (sítios I e II) + dominante (Sítio II) da população.

Figure 7. Line density of trees found in a stand of Hovenia dulcis in 25 years, belonging to the intermediary strata dominated + (sites I and II) + dominant (Site II) of the population.

Para esse caso, planeja-se aplicar quatro intervenções antes da ocorrência do corte raso, no fim da rotação. Todo o planejamento referente ao número de árvores remanescentes, área basal e o que será retirado nos desbastes é apresentado na tabela 6.

Tabela 6. Plano de aplicação de desbastes na condução de um povoamento de Hovenia dulcis com densidade de 1917 árvores/ha, em local com índice de sítio de 20 a 24 metros e idade de 25 anos.

Table 6. Plan application of thinning in the conduct of a stand of Hovenia dulcis with density of 1917 trees/ha on site with site index of 20 to 24 meters and 25 years of age.

\begin{tabular}{|c|c|c|c|c|c|c|}
\hline \multirow{2}{*}{$\mathrm{dg}(\mathrm{cm})$} & \multicolumn{2}{|c|}{ Remanescente } & \multicolumn{4}{|c|}{ Desbaste } \\
\hline & $\mathrm{N} / \mathrm{ha}$ & $\mathrm{G}\left(\mathrm{m}^{2} / \mathrm{ha}\right)$ & $\mathrm{N} / \mathrm{ha}$ & Peso (\%N/ha) & $\mathrm{G}\left(\mathrm{m}^{2} / \mathrm{ha}\right)$ & Peso $(\% \mathrm{G})$ \\
\hline 10,9 & 1917 & 17,89 & & & & \\
\hline 11,0 & 1300 & 12,35 & 617 & 32,19 & 5,53 & 30,94 \\
\hline 13,9 & 1300 & 19,73 & & & & \\
\hline 14,0 & 900 & 13,85 & 400 & 30,77 & 5,87 & 29,77 \\
\hline 16,9 & 900 & 20,19 & & & & \\
\hline 17,0 & 600 & 13,62 & 300 & 33,33 & 6,57 & 32,54 \\
\hline 22,9 & 600 & 24,71 & & & & \\
\hline 23,0 & 390 & 16,20 & 210 & 35,00 & 8,51 & 34,43 \\
\hline 31,0 & 390 & 29,43 & & & & \\
\hline
\end{tabular}

Segundo o planejado para esse caso, o povoamento será conduzido com a aplicação de uma série de 4 desbastes de intensidades moderadas, retirando-se o equivalente a 30 a $35 \%$ da área basal ou do número de árvores por hectare em cada intervenção. Especificamente para o caso em análise, tem-se a necessidade técnica da aplicação de várias intervenções, para não abrir demasiadamente o povoamento.

$\mathrm{Na}$ figura 8 é apresentada graficamente a demonstração da aplicação das intervenções sobre o Diagrama de manejo da densidade de estoque elaborado por Selle (2009) para a espécie e local. A linha 
oscilante contínua representa a primeira situação (um desbaste) e a linha oscilante tracejada refere-se à simulação da aplicação dos quatro desbastes da segunda simulação.

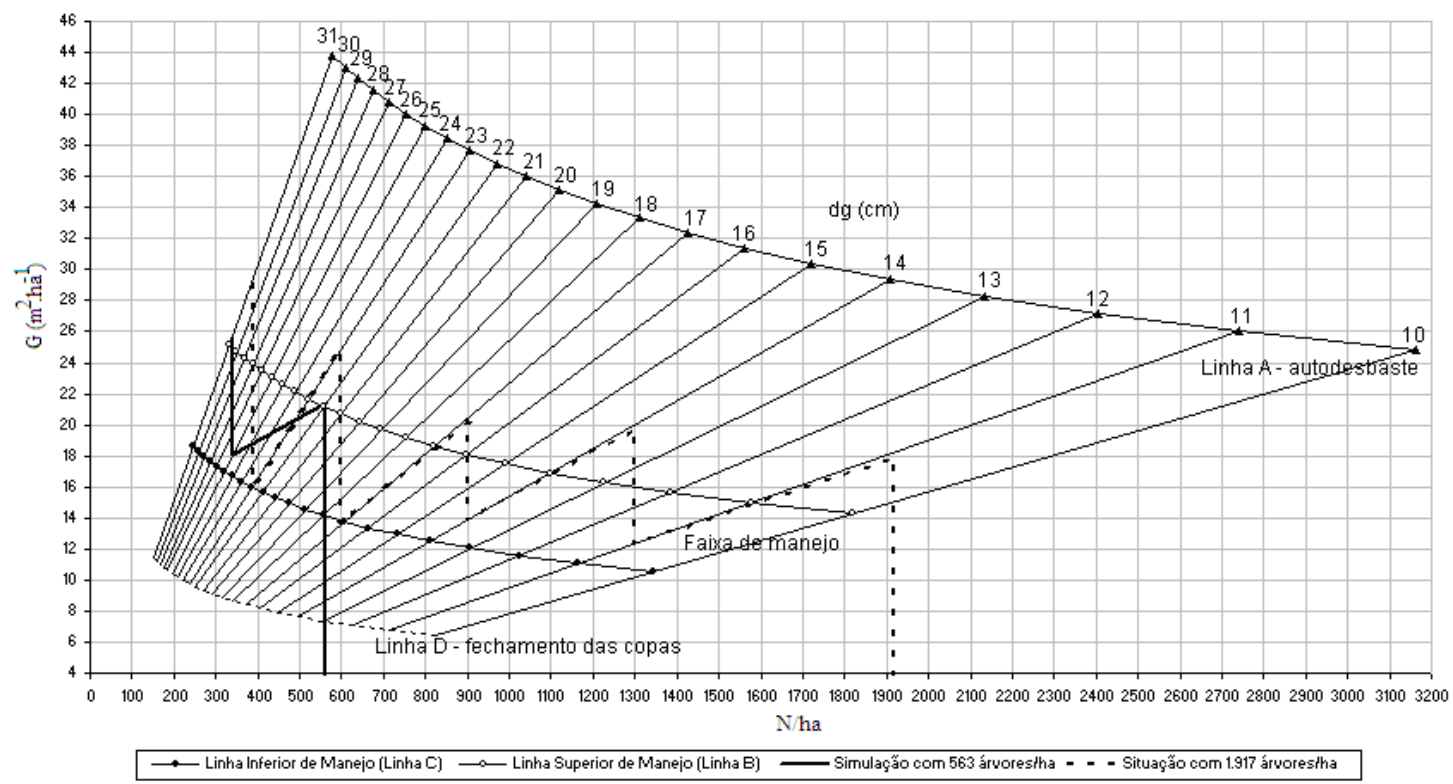

Figura 8. Simulação de aplicação dos desbastes sobre o diagrama de manejo de densidade para Hovenia dulcis.

Figure 8. Simulation for the application of thinnings on the density management diagram for Hovenia dulcis.

Observa-se que as intervenções extrapolaram a linha delimitadora superior da faixa recomendada de manejo (linha B), estabelecida por Selle (2009) em 57,5\% da linha de máxima densidade, num diâmetro padrão de $25 \mathrm{~cm}$, percentual este citado por grande parte da literatura sobre o assunto. No entanto essa linha foi estabelecida por um meio alternativo, já que não havia povoamentos da espécie em estágio de autodesbaste, caso mais indicado para o ajuste da linha, motivo pelo qual acredita-se que a linha projetada seja inferior à de autodesbaste real.

\section{CONCLUSÕES}

- O estudo das relações entre o diâmetro da copa e o diâmetro do tronco indicou haver diferenças estatísticas significativas entre sítios e estratos fitossociológicos, sendo necessária a análise em separado.

- Entre os estratos, ficou estabelecido que somente ocorreu diferença estatística significativa no diâmetro de copa entre o estrato dominante do Sítio I e os estratos intermediário e dominado dos sítios I e II, acrescido do dominante do Sítio II.

- Na simulação, ficou estabelecido que a melhor forma de conduzir o povoamento estudado variou com o sítio e o estrato fitossociológico, aplicando-se um desbaste, se ele fosse formado somente por árvores com dimensões semelhantes às do estrato dominante do Sítio I, e quatro intervenções se fosse constituído somente de indivíduos pertencentes aos demais estratos.

\section{AGRADECIMENTOS}

À Fundação de Pesquisa Agropecuária (FEPAGRO), por ter cedido a área para a realização da pesquisa, e ao Programa de Pós-Graduação em Engenharia Florestal da Universidade Federal de Santa Maria, por possibilitar a efetivação da pesquisa. 


\section{REFERÊNCIAS}

ASSMANN, E. The principles of forest yield study. New York: Pergamon Press, 1970. 506 p.

CARVALHO, P. E. R. Ecologia, silvicultura e usos da uva-do-japão (Hovenia dulcis Thunberg). Colombo: EMBRAPA Florestas, 1994. p. 24-65 (Circular Técnica, 23).

CHAPMANN, H. H.; MEYER, W. H. Forest mensuration. New York: McGrow Hill, 1949. 522 p.

DANIELS, R. F.; BURKHART, H. E.; CLASON, T. R. A comparison of competition measures for predicting growth of loblolly pine trees. Canadian Journal for Forest Research, Ottawa, v. 16, n. 6, p. 1230-1237, Dec. 1986.

DREW, T.; FLEWELLING, J. W. Stand density management: an alternative approach and its application to Douglas fir plantations. Forest Science, Bethesda, v. 25, n. 3, p. 518-532, Jun. 1979.

DURLO, M. A. Relações morfométricas para Cabralea canjerana (Well.) Mart. Santa Maria. Ciência Florestal, Santa Maria, v. 11, n. 1, p. 141-149, jan.jun. 2001.

FINGER, C. A. G. Fundamentos de biometria florestal. Santa Maria: CEPEF/FATEC/UFSM, 1992. 269 p.

GEZAN, S. A.; ORTEGA, A.; ANDENMATTEN, E. Diagramas de manejo de densidad para renovales de roble, raulí y coigüe em Chile. Bosque, Valdivia, v. 28, n. 2, p. 97-105, jul./dic. 2007.

GINRICH, S. R. Measuring and evaluating stocking and stand density in upland hardwood forests in the central states. Forest Science, Bethesda, v. 13, n. 1, p. 28-53, Feb. 1967.

HUSCH, B.; MILLER, I. C; BEERS, T. W. Forest mensuration. New York: J. Wiley, 1982. 402 p.

MALUF, J. R. T. Nova classificação climática do estado do Rio Grande do Sul. Revista Brasileira de Agrometeorologia, Santa Maria, v. 8, n. 1, p. 141-150, jan. 2000.

MÁRQUEZ-LINARES, M. A.; ALVAREZ-ZAGOYA, R. Construcción de uma guía de densidad para Pinus cooperi var. ornelasi com base en la clase de copa em Durango, México. Madera y Bosques, Xalapa, v. 1, n. 2, p. 23-36, May./Sep. 1995.

MICROSOFT ${ }^{\circledR}$ OFICCE EXCEL. Parte do produto Microsoft Office Professional 2003. Copryght $\odot$ 1985-2003 Microsoft Corporation. 2003.

PARVIAINEN, J.; NAUMANN, N. A Finlândia e suas florestas. Revista Floresta, Curitiba, v. 1/2, n. 16, p. 5-18, jan./dez. 1986.

PEET, R. K.; CRISTENSEN, N. L. Composition and tree death. BioScience, Washington, v. 37, n. 11, p. 586-681, Nov. 1987.

REINEKE, L. H. Perfecting a stand-density-index for even aged forests. Journal of Agricultural Research, Beltsville, v. 7, n. 46, p. 627-638, Jan./Dec. 1933.

RIGATTO, P. A.; PEREIRA, J. C. D.; MATTOS, P. P. de; SCHAITZA, E. G. Características físicas, químicas e anatômicas da madeira de Hovenia dulcis. Colombo: EMBRAPA Florestas, 2001. 4 p. (Comunicado Técnico, 66).

RIGOZO, N. R.; NORDEMANN, D. J. R. Registros da atividade solar nos anéis de crescimento de árvores em São Francisco de Paula, RS, Brasil. Brazilian Journal of Geophysics, São José dos Campos, v. 18, n. 1, p. 91-97, mar. 2000.

SAS. The SAS System for Windows. Copyright (c) 1999-2001 by SAS Institute Inc., Cary, NC, USA. 1999.

SAUNDERS, M. R.; PUETTMANN, K, J. A preliminary white spruce density management diagram for the lake states. Minnesota: University of Minnesota. College of Natural Resources and Minnesota Agricultural Experiment Station. Department of Forest Resources. 2000. 14 p. (Sraff Paper Series, n.145). 
SCHEEREN, L. W. Estruturação da produção de provoamentos monoclonais de Eucalyptus saligna Smith manejados em alto fuste. $181 \mathrm{f}$. Tese (Doutorado em Engenharia Florestal) - Universidade Federal de Santa Maria, Santa Maria, 2003.

SCHNEIDER, P. R. Introdução ao manejo florestal. Santa Maria: CEPEF/FATEC, 1993. 348 p.

SCHNEIDER, P. S. P. Autodesbaste e diagrama de manejo da densidade em povoamentos de Pinus taeda L. 94 f. Dissertação (Mestrado em Enganharia Florestal) - Universidade Federal de Santa Maria, Santa Maria, 2008.

SELLE, G. L.; OLIVEIRA, O. dos S.; CAMPANHOL, E. L. R.; ZIMMERMANN, A. Influência de diferentes tipos de cobertura na germinação e sobrevivência de mudas de uva-do-japão. Agropecuária Catarinense, Florianópolis, v. 6, n. 4, p. 20-22, nov. 1993.

SELLE, G. L. Guias de densidade e índices de sítios para Holvenia dulcis Thunberg na Região Central do Estado Rio Grande do Sul, Brasil. 97 f. Tese (Doutorado em Engenharia Florestal) Universidade Federal de Santa Maria, Santa Maria, 2009.

SELLE, G. L.; VUADEN, E. Comportamento do mercado de produção e do consumo de madeira serrada. Caderno de Pesquisa. Série Biologia, Santa Cruz do Sul. 2009. No prelo.

SMITH, C.; GIBBS, C. B. A guide to sugarbusch stocking: based on the crown diameter/D.b.h. relationship of open-grown sugar maples. Washington: Forest Service, 1970. 10 p. (Reserarch Paper NE-171).

SPIECKER, H. Análise do crescimento florestal: a concorrência e sua importância no desbaste. Curitiba: FUPEF, 1981. 62 p. (Série Técnica FUPEF, n.8).

SPURR, S. H. A measure of point density. Forest Science, Bethesda, v. 8, n. 1, p. 85-96, Feb. 1962.

SPURR, S. H. Forest Inventory. New York: The Ronald Press Company, 1952. 476 p.

U.S. FOREST SERVICE. Relation of crown width to tree diameter in same upland hardwood stands of southern Illinois. St. Paul: Southern Forest Experiment Station Library. 1970. 4 p. (Research Note NC-99).

YODA, K.; KIRA, T.; OGAWA, H.; HOZUMI, K. Self-thinning in over rowed pure stands under cultivated and natural conditions. Journal of Biology, London, v. 14, n. 1., p. 107-129, Jan./Dec. 1963. 